\title{
KenVACS: Improving Vaccination of Children through Cellular Network Technology in Developing Countries
}

\author{
George Gatuha and Tao Jiang \\ College of Information and Communication Engineering, \\ Harbin Engineering University, Heilongjiang, China
}

\section{ggrpb@yahoo.com jiangtao@hrbeu.edu.cn}

\begin{abstract}
Health Data collection is one of the major components of public health systems. Decision makers, policy makers, and medical service providers need accurate and timely data in order to improve the quality of health services. The rapid growth and use of mobile technologies has exerted pressure on the demand for mobile-based data collection solutions to bridge the information gaps in the health sector. We propose a prototype using open source data collection frameworks to test its feasibility in improving the vaccination data collection in Kenya. KenVACS, the proposed prototype, offers ways of collecting vaccination data through mobile phones and visualizes the collected data in a web application; the system also sends reminder short messages service (SMS) to remind parents on the date of the next vaccination. Early evaluation demonstrates the benefits of such a system in supporting and improving vaccination of children. Finally, we conducted a qualitative study to assess challenges in remote health data collection and evaluated usability and functionality of KenVACS.
\end{abstract}

Keywords: Health Data collection, Short Message Service, Open Source data frameworks, Mobile Technologies, Vaccination, Kenya.

\section{Introduction}

Vaccination has been identified as the most effective method of reducing the burden of infectious diseases (Andre et al, 2008); the worldwide eradication of small pox is one of the benefits of vaccination and the restriction of the spread of diseases such as tetanus, measles, and polio from much of the world. The World Health Organization (2014) began an ambitious campaign for the period 2011-2020 that is aimed at delivering vaccines to all and to unleash vaccines' vast future potential.

Material published as part of this publication, either on-line or in print, is copyrighted by the Informing Science Institute. Permission to make digital or paper copy of part or all of these works for personal or classroom use is granted without fee provided that the copies are not made or distributed for profit or commercial advantage AND that copies 1) bear this notice in full and 2) give the full citation on the first page. It is permissible to abstract these works so long as credit is given. To copy in all other cases or to republish or to post on a server or to redistribute to lists requires specific permission and payment of a fee. Contact Publisher@,InformingScience.org to request redistribution permission.
To eliminate the risk of outbreaks of vaccine preventable diseases, several governments and other institutions have instituted policies that require vaccination of all people and especially the chilchildren. A good example is a British law passed in 1853 that required everybody to be vaccinated against smallpox in Britain, with non-compliant people levied fines (Craig, 2010). In the United States, contemporary vaccination poli- 
cies require that all children receive common vaccinations before enrollment in public schools (Cole \& Swendiman, 2014). However, these policies are resisted by anti-vaccinationists, a civil society group who object to vaccination and other scientific interventions. Common objections are that vaccinations do not work, that compulsory vaccination represents excessive government intervention in personal matters, or that the vaccinations are not sufficiently safe (Wolfe \& Sharp, 2002).

Parents and guardians are concerned about the safety and health of their children. Therefore they take great precautions to protect their children from being sick; it is every parent's wish for their children to be free of any sickness. To prevent children from getting sick, vaccination is one of the best options. However there are schedules to be adhered to for vaccination to be effective and the schedules vary from adult to children (Lance, 2005).

Vaccination reminder and recall systems are cost-effective methods whereby children in need of vaccination are identified and contacted to come to the health centers to receive vaccination. Reminder systems can work through a variety of mechanisms meant to prompt the patient, including media campaigns, telephone, posters, e-mail, and short message service (SMS). While all types of reminder systems are effective, telephone reminders have been found to be the most ideal, but also the most expensive compared to other mediums (Szilagyi et al., 2000).

Short Message Service (SMS) is a text messaging service component of mobile communication systems. SMS was the most widely used data application by about $80 \%$ of all mobile phone subscribers at the end of 2010 (Tomi, 2011). SMS permits users to communicate non-verbally, through coalitions of alphanumerical symbols with a maximum of 160 characters per SMS message. SMS has become popular globally because SMS is a relatively cheap, fast and efficient means of connection between people of any distance (Soriano, Gitesh, \& Szajman, 2005).

Kenya has undergone a remarkable information and communications technology revolution. At the end of the last millennium, about three percent of Kenyan households owned a telephone, and less than $0.1 \%$ of Kenyan adults had cell phone connectivity. By the end of 2013, $93 \%$ of Kenyan households owned a mobile phone. According to the latest statistics report published by the Communication Authority of Kenya (CAK) (2013), the country's mobile penetration rate is $77.3 \%$, representing about 30.5 million people. The level of penetration is still persistently below the global (91.2\%), developed (123.6\%), and developing countries' (84\%) penetration rates as of December 2012. Developing and implementing an SMS application reminder system would therefore reach a large number of people.

\section{Related Work}

Many ICT interventions have been introduced to address health challenges including those of rural communities. Rohit and Borriello (2010) designed a mobile phone based system (FoneAstra) for remote monitoring of the temperature and location of vaccines. The system is equipped with a temperature sensor and integrated with a vaccine cold-box that is used to store vaccines in a temperature regulated environment. FoneAstra monitors on a regular basis the temperature of the cold-box, aggregating the readings over time. It uses a mobile phone, to which it is coupled, to send periodic SMS messages with routine temperature reports or immediate alerts if it detects temperature above the threshold. Additionally, it enables location-tracking of vaccines on transit, based on the mobile phone's Wi-Fi.

Zhang, Wang, Liu, and Hou (2006) designed a system for fall detection using off-the-shelf electronic devices to detect the fall. They used a smart phone with an embedded tri-axial acceleration sensor. Information from the sensor is evaluated with a decision tree model to determine whether a fall has taken place. If a fall is recorded, a notification is raised to require the user's response. If 
the user's body is hurt and unable to respond, an alert via SMS is sent to a guardian. Therefore, the fallen individual can be cared for immediately.

Anas, Alaa, Chulawadee, and Adib (2011) designed a web-based system to ensure pregnant mothers are notified regarding their pregnancy progress via SMS message.

Osama (2012) developed a mobile phone SMS-based system for diabetes self-management. The system allows the patient to be connected to his or her physician and send insulin measurements, insulin intake, and other vital data, making continuous real time health monitoring possible. The physician also sends motivational SMSs or reminders to the patients to do some exercises and attend health care appointments.

United Nations Foundation (2010) developed a Rural Extended Services and Care for Ultimate Emergency Relief (RESCUER) application that combined use of two way radios with ambulances and bicycles to adequately respond to obstetric emergencies, which resulted in a $50 \%$ reduction in mortality rates of women in Uganda.

Sherwani et al. (2007) developed a speech-based health information access system called Health Line for low literate community health workers in the Sindh Province of Pakistan. The community health workers were unable to read at all or did with a lot of difficulty. The system uses an instructor to guide the health workers in the field.

\section{Design and Development}

KenVACS system is meant to assist clinicians in collecting patient demographic and vaccination information. The information is stored in a small in-built SQLite data base and later transferred to an online portal through Wi-Fi. Patients are prompted through SMS on their next vaccination date automatically.

In Kenya health data flows from the health centers to the district, then to the provincial headquarters, and finally to the national level. The data is collected manually using paper and pen and rarely collected using computerized methods such as MS Excel programs or other spread sheets. At the primary health centre or dispensary, patient data is collected using small paper cards or an exercise book and which is later transferred to a register. The registers are used to generate tally's which are later compiled into forms referred to as "book A". The forms are then transferred to the district where they are compiled and new forms, called "book B," are generated. Book B forms are finally submitted to the Ministry of Health headquarters for record keeping and analysis.

\section{Stakeholder Identification}

The identification of stakeholders plays an important role in software development because it guides the engineering process. The stakeholders were identified using guidelines from the Ministry of Health.

Health officers: These are experts at the health centers whose duties are to attend to patients and any other health related duty. These officers, apart from offering treatment, will also collect the patient information through electronic forms using their mobile phones.

Health system managers: These are the decision and policy makers at the Ministry Headquarters who are tasked to visualize the data, analyze and make policy decisions geared towards the improvement of health services.

Parents / guardians: they will receive SMS alerts about forthcoming vaccination campaigns as well as query the system about vaccination status of their children. 
Functional requirements: These are the specific statements that describe how the system should react to inputs and its general behavior in certain situations; this corresponds to the system expectations.

F1. The backend module of the prototype provides ways of visualizing and analyzing the data.

Motivation: Data analysis is important for decision and policy makers.

F2. Client side of the prototype shall provide forms for gathering data using a mobile phone.

Motivation: Among the key features that qualify a mobile phone as an ideal data collection tool is its portability, ability to store charge, and its usability especially in remote areas where electric power is unavailable. The proposed prototype shall provide a medium for data collection using forms and synchronize to a central database through Wi-Fi.

F3. The parents or guardians shall be able to download data from the central server. They will also be able to receive alerts on health related issues and a reminder for next vaccination date.

Motivation: Centralization of data will bridge the gap of data fragmentation and ease of acquisition that is common to paper based manual data collection techniques.

\section{Design of the Prototype}

The prototype was designed using an open source mobile Operating System (OS). Android is based on the popular Linux OS, Dalvik Virtual Machine and Apache harmony, and is headed by Google, and has recently gained tremendous market share and popularity among developers and end-users (Gandhewar \& Sheikh, 2011). We arrived at the decision to use Android OS due to its fast growth and de facto acceptance as well as openness in its operating system and Application Programming Interfaces (APIs). The prototype was developed in the Android platform using the object-oriented Android programming paradigm and Android Software Development Kit (SDK). The business logic was implemented using activities, the special case Android classes, while the Graphical User Interface was designed and implemented using the Extensible Markup Language (XML). Figure 1 depicts the Android development architecture.

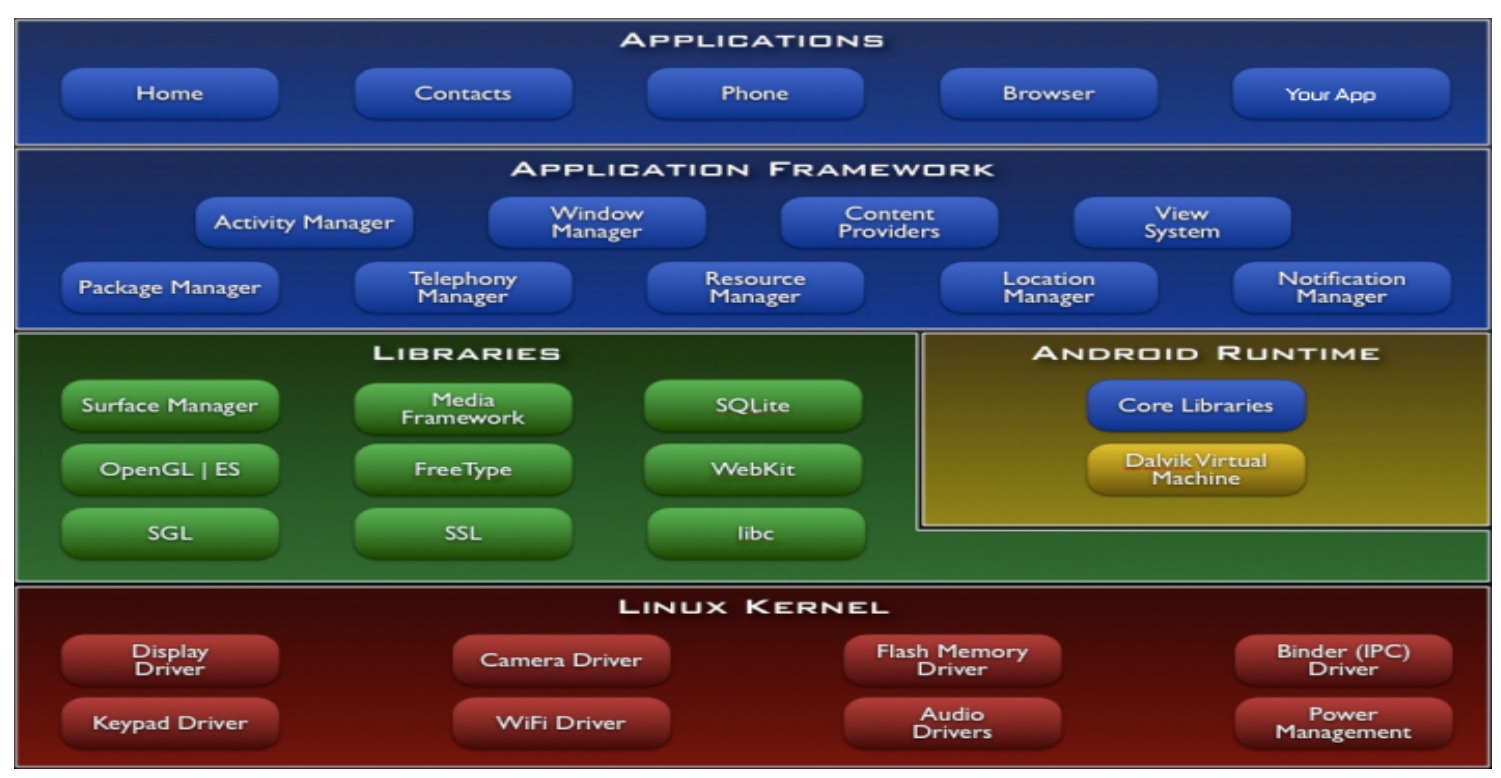

Figure 1: The Android development architecture 
Personal Home Page (php), an open source server-side scripting language designed for web development, was used to enable seamless and secure data transfer between the android application and the MySQL database. The program was originally developed by Rasmus Lerdorf ("History of PHP", 2014), however the reference implementation of php is now produced by The PHP Group.

FrontlineSMS is an open source software package developed by Kiwanja.net under GNU license as an SMS gateway (Banks, 2007); the software was used in our project to send reminder messages to parents. The software can work without internet connection and with only a GSM (Global System for Mobile Communications) or a GPRS (General Packet Radio Service) modem and a computer (Gow \& Waidyanatha, 2010). External commands were developed using php that enabled the FrontlineSMS system to automatically send messages to parents.

MySQL is an open source relational database management system and is used to store our vaccination records. Although Android platform's native database is SQlite, the syntax of the queries is similar to MySQL, and php scripts were developed in order to create the appropriate data format so that the required data will be served correctly to the database through a hypertext transfer protocol (HTTP) connection. All the services are offered through the Apache server.

Eclipse IDE was used to develop the Android application. The main objective was to achieve the local storage of data in a small SQLite database and upload the data to the Server via the internet. However, this proved to be the most challenging part since the platform does not yet support the connection through a driver [e.g., ODBC driver] to a remote database. Thus, it had to be implemented from scratch. The most convenient way was the implementation of php scripts that accept arguments through HTTP post and display the results on the MySQL database tables. To achieve this, a function was implemented so that Android can send data through HTTP post and receive the response from the server.

The architecture of KenVACS system is based on a server-client connection model. The centralized server, located at the Ministry headquarters will provide to the client (a mobile handheld device - e.g., a mobile phone or tablet), patient data storage, and the server will also provide data to the patient upon request. In Figure 2, the KenVACS system architectural design is presented.

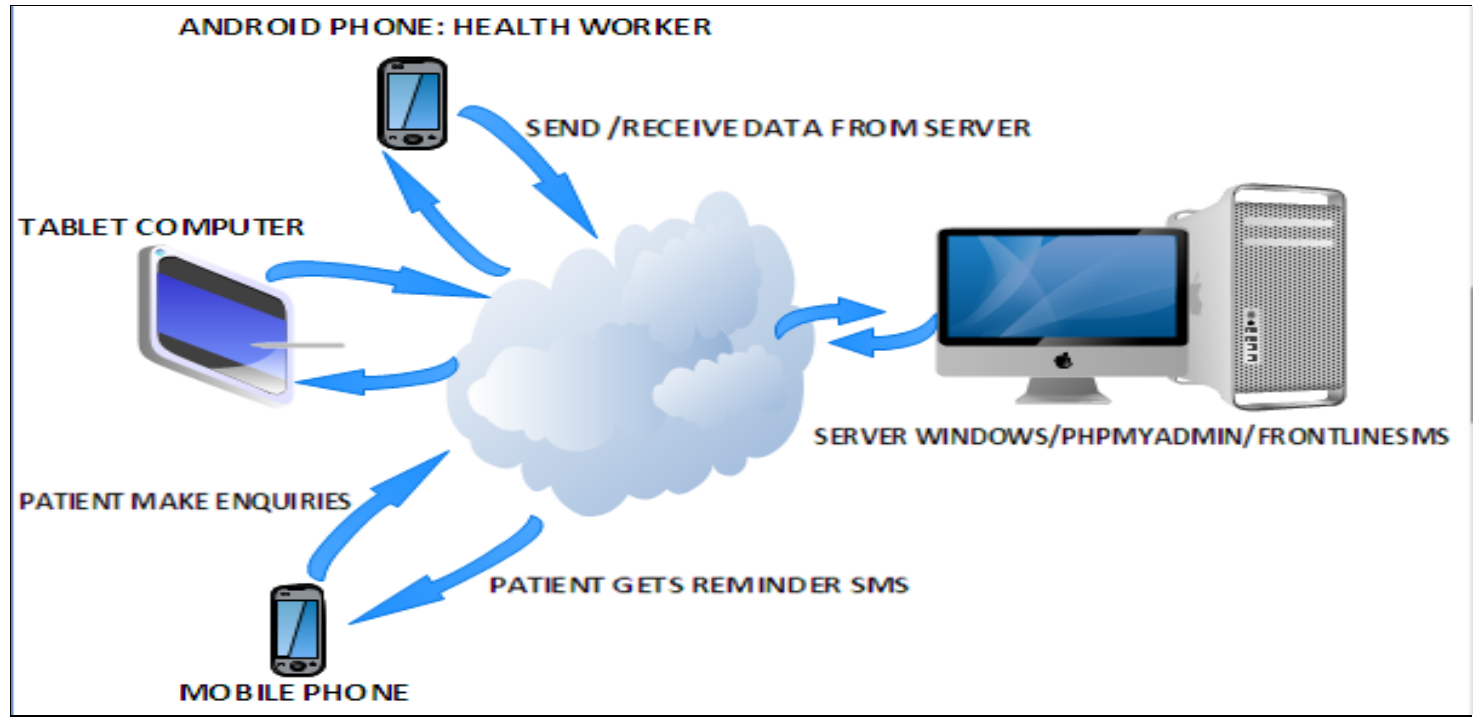

Figure 2. KenVACS system architectural design

Workflow: Health staff registers details of the new born children in the system. This is done using the Android tablet or a mobile phone. The health personnel must capture the demographic details of the child such as in Figure 3 (patient ID, names, gender, mothers name, date of birth, and con- 
tact details of parents) and store the information in the phone's SQLite database; due to the limitations on internal phone's memory; once the records are uploaded to the central server, they are automatically deleted from the phone's SQLite database.

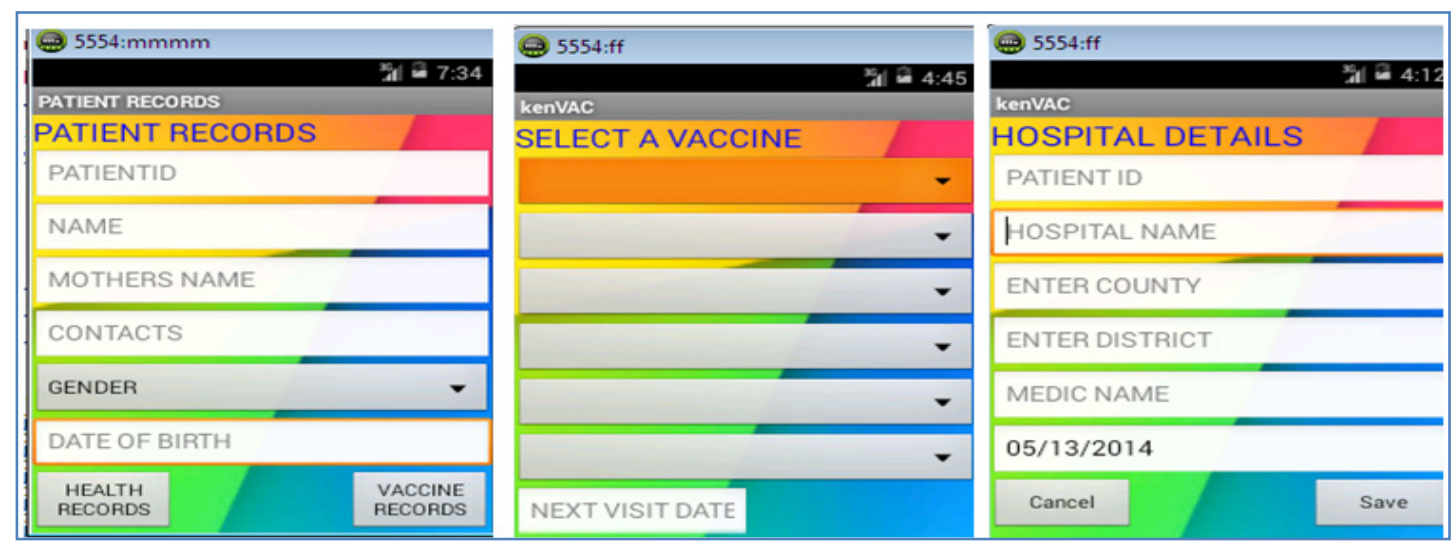

Figure 3: GUI of the mobile application

After successful update of information in the MySQL Database (Figure 4), the system continuously checks the database to produce a report of which children are due to be vaccinated in a certain day and automatically sends the parents/guardian a short message text (SMS) reminder a day before the due date. After vaccination and with sufficient data the health personnel update the central database system as appropriate. The parents may also enquire on vaccination status of the child by sending an SMS to a prescribed telephone number using a key word such as vaccine, name of the child and phone number.

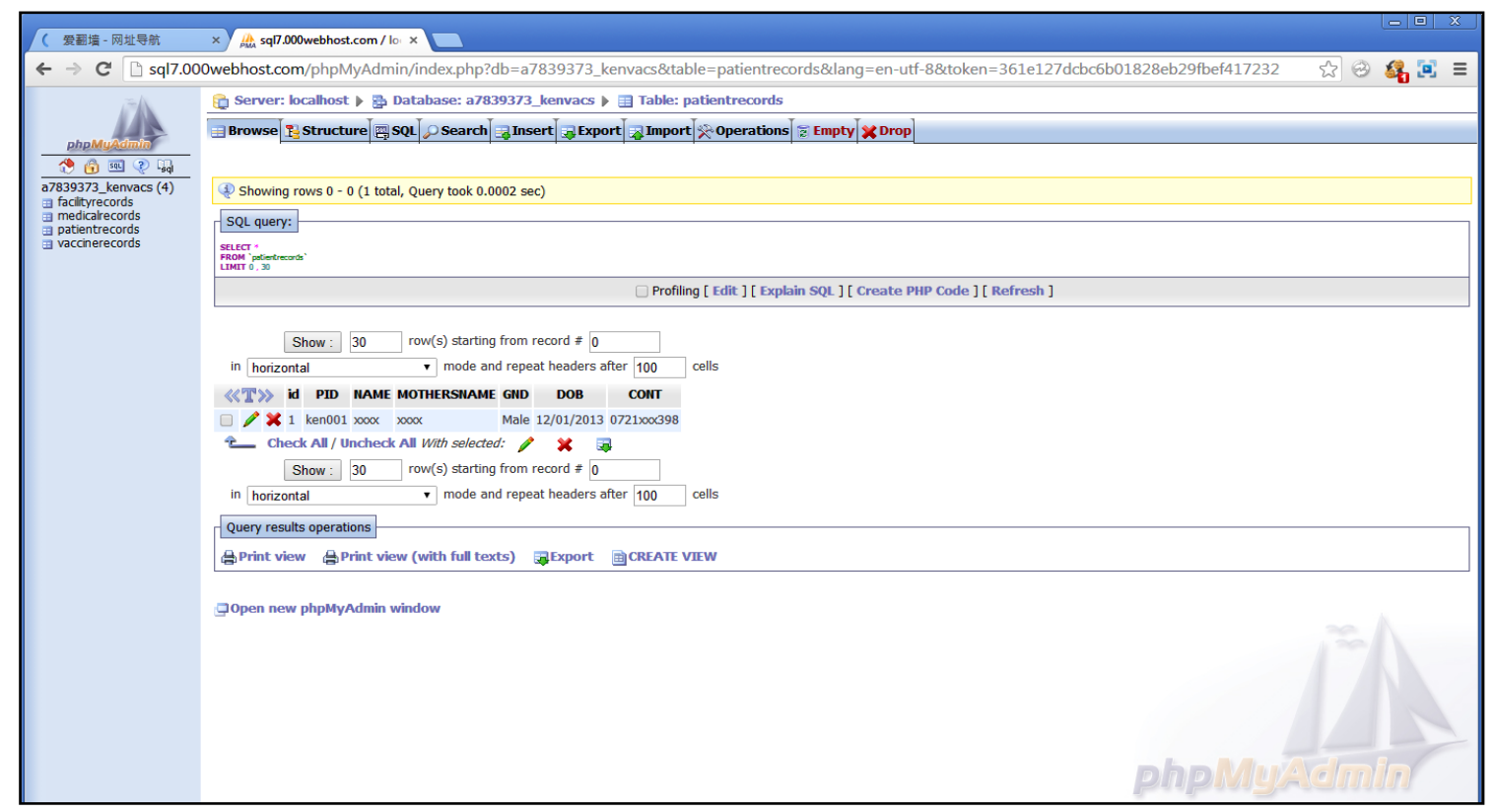

Figure 4: MySQL database

\section{Results and Analysis}

The Server-Client system was evaluated using the developed software, in an Android simulation environment, in real time (using a desktop PC with access to a wireless connection and an Android based mobile phone), and finally tested on a web server. Apart from the operational evalua- 
tion, a functional and usability evaluation was also conducted. We faced several issues, like the development of API's to enable FrontlineSMS to get information from the database and automatically send SMS to patients.

After the evaluation of the server-client connection, the developed prototype was then tested at Kajiado County Hospital, a rural hospital in Kenya. A total of 33 respondents was used to evaluate the prototype.

Analysis of the respondents showed that 18 have two children (54.5\%), while 10 have one child $(30.3 \%)$, and five of them (15.2\%) have more than two children. The respondent's number of children is shown in Table1.

\begin{tabular}{|l|c|l|}
\hline \multicolumn{3}{|c|}{ Table 1: Respondents number of children } \\
\hline CHILDREN & FREQUENCY & PERCENTAGE (\%) \\
\hline 1 child & 10 & 30.3 \\
\hline 2 children & 18 & 54.5 \\
\hline$<$ children & 5 & 15.2 \\
\hline TOTAL & $\mathbf{3 3}$ & $\mathbf{1 0 0 . 0}$ \\
\hline
\end{tabular}

The majority of the respondent's ages was found to be below 40 years old as given in Table 2 .

\begin{tabular}{|c|c|c|}
\hline \multicolumn{3}{|c|}{ Table 2: Respondents age group } \\
\hline AGE & FREQUENCY & PERCENTAGE (\%) \\
\hline $20-30$ & 14 & 42.4 \\
\hline $31-40$ & 16 & 48.5 \\
\hline+40 & 3 & 9.1 \\
\hline TOTAL & $\mathbf{3 3}$ & $\mathbf{1 0 0 . 0}$ \\
\hline
\end{tabular}

The undertaken evaluation also reveals that $63.6 \%$ of the respondents sometimes forget their child's vaccination date (Table 3 ).

\begin{tabular}{|l|c|c|}
\hline \multicolumn{3}{|c|}{ Table 3: Respondents ability to recall schedule } \\
\hline PERCEPTION & FREQUENCY & PERCENTAGE (\%) \\
\hline Always & 3 & 9.1 \\
\hline Sometimes & 21 & 63.6 \\
\hline Never & 9 & 27.3 \\
\hline TOTAL & $\mathbf{3 3}$ & $\mathbf{1 0 0 . 0}$ \\
\hline
\end{tabular}

It was also discovered (Table 4) that parents do agree on having a reminder to be sent to their mobile phone. A total of 18 respondents observed that the system is very useful, while 15 found it to be useful as an alert mechanism for reminding them the date of vaccination appointment.

\begin{tabular}{|l|c|c|}
\hline \multicolumn{3}{|c|}{ Table 4: Respondents view of SMS usefulness. } \\
\hline PERCEPTION & FREQUENCY & PERCENTAGE (\%) \\
\hline Very useful & 18 & 54.5 \\
\hline Useful & 15 & 45.5 \\
\hline Not useful & 0 & 0.0 \\
\hline Strongly not useful & 0 & 0.0 \\
\hline Total & $\mathbf{3 3}$ & $\mathbf{1 0 0 . 0}$ \\
\hline
\end{tabular}


The response on the proposed alert system was very impressive; all of the respondents agreed that the proposed approach of sending a vaccination schedule reminder via SMS is ideal.

Of the 33 respondents $78.8 \%$ preferred to be reminded through SMS while $21.2 \%$ preferred the child immunization card for reference (Table 5).Such results suggest the importance of this study and the possibility of its application in the health sector.

\begin{tabular}{|l|c|c|}
\hline \multicolumn{3}{|c|}{ Table 5: Respondents preferred mode of reminder. } \\
\hline MODE OF REMIND- & FREQUENCY & PERCENT (\%) \\
\hline Via SMS & 26 & 78.8 \\
\hline Child Immunization card & 7 & 21.2 \\
\hline Other means & 0 & 0.0 \\
\hline Total & $\mathbf{3 3}$ & $\mathbf{1 0 0 . 0}$ \\
\hline
\end{tabular}

The subjective evaluation showed that system user experience was smooth and realized with satisfaction.

\section{Conclusion}

In this paper we have outlined the use of a mobile communication application for improving vaccination data collection, implementation of an SMS vaccination reminder system, and outlined the benefits of a centralized health data management system. Through the relevant literature review we have done an analysis of current methods employed in health data collection that have led to improved health service delivery. KenVACS focused on health data collection in remote areas where computing resources are very few by utilizing mobile phone handsets of health officers. The overall aim is to improve health services by digitizing data collection and enabling exchange of information between the patient and the health workers, with a special focus in developing countries. The system will definitely improve uptake of vaccination health services and data management.

The system was developed using the Android platform and Eclipse integrated development environment. The developers arrived at this decision based on their ease of use, lack of purchase price, and the massive freeware community. After careful evaluation, the client server design was found to offer the best platform for data exchange between the client and the system administrators. Patients can get real-time access to medical vaccination records and vaccination reminders, while the health workers benefit from efficiency and time-saving data collection methods provided by KenVACS.

KenVACS represents a step closer in bridging the gap between analog and digital data analysis in the health sector realms. The system will enable the developing world to reap benefits related to the positive impact of augmenting a doctor's diagnosis and information technology benefits. There is a serious lack of this technology in the health sector of the developing world, and KenVACS attempts to addresses that gap.

Some planned features were shelved for future development. The most important of these is to provide patients with the ability to store and access their own health information without participation of the clinician. However, the usability of this technology would greatly benefit from lowering of internet connection costs which are prohibitively high in the developing world and Africa in general. 
Future studies could also be geared towards improving on health data security. Rapid advancement of ICT's increases vulnerability of the privacy and security of health data, especially sensitive health data which might have great impact on the health sector. Following in the footsteps of social media platforms, health platforms in similar directions could impact positively on linking people affected by similar diseases and exchanging ideas on best treatment regimens and cost benefits that may be associated with some health issues.

The system can also be easily customized for use in other sectors unrelated to healthcare such as the registration of persons, development of banking applications, student registration, and checking of price fluctuations in agricultural market, among many other areas.

Moreover, there is a need to investigate how open source frameworks such as data mining techniques could enhance other health management services especially in the developing world.

\section{Acknowledgements}

All KenVACS modules were developed in collaboration with the College of Information and Communication Engineering, Harbin Engineering University, China. The prototype was tested at the Ministry of Health, Kajiado County Hospital, Kenya. The study was actualized through the generous assistance of both the Kenyan and the Chinese governments.

\section{References}

Anas, S., Alaa, A., Chulawadee, K., \& Adib, M. (2011). Web based support for pregnant mother. International Journal on Advanced Science, Engineering and Information Technology, 1(4), 307-310.

Andre, F. E., Booy, R., Bock, H. L., Clemens,J., Datta, S. K., John,T. J., Lee, B. W., ...Schmitt, H. J. (2008). Vaccination greatly reduces disease, disability, death and inequity worldwide. Retrieved August 27, 2014, from http://www.who.int/bulletin/volumes/86/2/07-040089.pdf

Banks, K. (2007).The story of FrontlineSMS. SAUTI, Stanford Journal of African Studies, IV, 2-4. Retrieved July 13, 2014, from http://www.stanford.edu/group/sauti/Archives_files/SAUTI-0708\%20Vol\%204.pdf

Cole, J. P., \& Swendiman, K. S. (2014). Mandatory vaccinations: Precedent and current laws. Retrieved August 20, 2014, from https://www.fas.org/sgp/crs/misc/RS21414.pdf

Communication Authority of Kenya. (2013). Annual report 2012 -2013. Retrieved August 4, 2014, from http://www.cck.go.ke

Craig, J. (2010).Smallpox vaccine: Origins of vaccine madness. Retrieved August 20, 2014, from http://www.vaccinationcouncil.org/2010/02/26/smallpox-vaccine-origins-of-vaccine-madness

Gandhewar, N., \& Sheikh, R. (2011). Google android: An emerging software platform for mobile devices. International Journal on Computer Science and Engineering, 12-17.

Gow, G. A., \& Waidyanatha, N. (2010) .Using mobile phones in a real-time bio surveillance program: Lessons from the FrontlineSMS in Sri Lanka and India. IEEE International Symposium on Technology and Society, 7-9 June, Wollongong, Australia. 366 - 374.

History of PHP. (2014). Retrieved August 21, 2014, from http://php.net/manual/en/history.php.php

Lance, E. R. (2005). Childhood immunization. Retrieved August 21, 2014, from http://www.childencyclopedia.com/documents/RodewaldANGxp.pdf

Osama, S. (2012) .A mobile phone SMS-based system for diabetes self-management. Retrieved August 21, 2014, from www.iajet.org/.../A\%20Mobile $\% 20$ Phone $\% 20$ SMS-Based $\% 20$ System $\% 2$

Rohit, C., \& Borriello, G. (2010). FoneAstra: Making mobile phones smarter. Retrieved August 11, 2014, From http://homes.cs.washington.edu/ rohitc/Publications files/NSDR $\% 202010 \% 20$ camera\%20ready.pdf 
Sherwani, J., Ali, N., Mirza, S., Fatma, A., Memon, Y., Karim, M., Tongia, R.,...Rosenfeld, R. (2007). HealthLine: Speechbased access to health information by low literate users. Proceedings of 2007 IEEE/ACM International Conference on Information and Communication Technologies and Development. pp. 131-139, Bangalore, India.

Soriano, C., Gitesh, K. R., \& Szajman, J. (2005). A usability study of short message service on middle-aged users. Retrieved July 11, 2014, from http://dl.acm.org/citation.cfm?id=1108443

Szilagyi, P. G., Bordley C., Vann, J. C., Chelminski, A., Kraus, R. M., Margolis, P. A,... Rodewald, L. E. (2000). Effect of patient reminder/recall interventions on immunization rates. Retrieved July 9, 2014, from http://jama.jamanetwork.com/issue. aspx $?$ journalid $=67 \&$ issueid $=4755$

Tomi, T. A. (2011). Time to confirm some mobile user numbers: SMS, MMS, Mobile Internet, M-News. Retrieved July 4, 2014, from http://communities-dominate.blogs.com/brands/2011/01/time-to-confirmsome-mobile-user-numbers-sms-mms-mobile-internet-m-news.html

United Nations Foundation. (2010). Mhealth for the developing world. Retrieved July 21, 2014, from http://www.unfoundation.org/assets/pdf/mobilizing-development-report.pdf

World Health Organization. (2014). Assessment report of the global vaccine action plan. Retrieved August 9, 2014, from http://www.who.int/immunization/global_vaccine action_plan/SAGE_DoV_GVAP_Assessment_repo rt 2014 English.pdf?ua $=1$

Wolfe, R., \& Sharp, L. (2002). Anti-vaccinationists, past and present. Retrieved July 13, 2014, from http://bmj.com/cgi/pmidlookup?view=long\&pmid=12193361

Zhang, T., Wang, J., Liu, P., \& Hou, J. (2006). Fall detection by embedding an accelerometer in cellphone and using KFD algorithm. Retrieved July 13, 2014, from http://www.scirp.org/journal/PaperInformation.aspx?PaperID=18069

\section{Biographies}

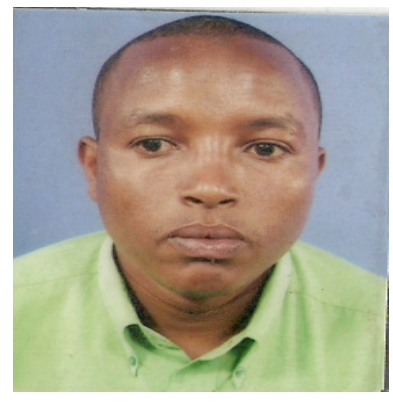

George Gatuha is a $\mathrm{PhD}$ candidate at the College of Information and Communication Engineering, Harbin Engineering University, China. His research interests include design and development of mhealth applications, design of NFC systems, and Data Engineering in Medicine. Member of International Association of Engineers (IAENG).

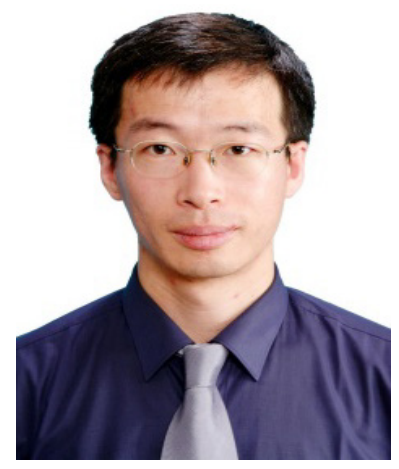

Tao Jiang is a Doctor of Engineering, Professor, Doctoral Supervisor at the College of Information and Communication Engineering, Harbin Engineering University, China. Senior member of China Institute of Communications, member of IEEE Harbin section EMC Society Chapter.

Research Area: Radio navigation and positioning, EMC theory and technology, Electromagnetic scattering and measurement. 